\title{
Malaria and related outcomes in patients with intestinal helminths: a cross-sectional study
}

\author{
Abraham Degarege*, Mengistu Legesse, Girmay Medhin, Abebe Animut and Berhanu Erko
}

\begin{abstract}
Background: The effects of helminth co-infection on malaria in humans remain uncertain. This study aimed to evaluate the nature of association of intestinal helminths with prevalence and clinical outcomes of Plasmodium infection.
\end{abstract}

Methods: A cross-sectional study involving 1,065 malaria suspected febrile patients was conducted at Dore Bafeno Health Center, Southern Ethiopia, from December 2010 to February 2011. Plasmodium and intestinal helminth infections were diagnosed using Giemsa-stained blood films and Kato-Katz technique, respectively. Haemoglobin level was determined using a haemocue machine.

Results: Among 1,065 malaria suspected febrile patients, 28.8\% were positive for Plasmodium parasites (P. falciparum $=13.0 \%$, P. vivax $=14.5 \%$, P. falciparum and P. vivax $=1.3 \%$ ). Among 702 patients who provided stool samples, 53.8\%, 31.6\% and 19.4\% were infected with intestinal helminths, Plasmodium alone and with both Plasmodium and intestinal helminths, respectively. The prevalence of infections with Ascaris lumbricoides (A. lumbricoides), Trichuris trichiura (T. trichiura), Schistosoma mansoni (S. mansoni) and hookworm (9.8\%) were 35.9\%, 15.8\%, $11.7 \%$ and $9.8 \%$, respectively. Out of the 222 (31.6\%) Plasmodium infected cases, 9 (4.1\%) had severe malaria. P. falciparum infection was more common in febrile patients infected with A. lumbricoides alone (21.3\%), T. trichiura alone (23.1\%) and S. mansoni alone (23.1\%) compared to those without intestinal helminth infections (9.3\%) $(p<0.001$ for all). Prevalence of non-severe malaria was significantly higher in individuals infected with intestinal helminths than in those who were not infected with intestinal helminths (adjusted $\mathrm{OR}=1.58,95 \% \mathrm{Cl}=1.13-2.22$ ). The chance of developing non-severe P. falciparum malaria were 2.6, 2.8 and 3.3 times higher in individuals infected with A. lumbricoides alone, T. trichiura alone and S. mansoni alone, respectively, compared to intestinal helminth-free individuals ( $p<0.05$ for all). The odds ratio for being infected with non-severe $P$. falciparum increased with the number of intestinal helminth species $(p<0.001)$. Mean Plasmodium density among intestinal helminth infected individuals was significantly increased with the number of intestinal helminths species $(p=0.027)$. Individuals who were co-infected with different species of intestinal helminths and Plasmodium showed lower mean haemoglobin concentration than individuals who were infected only with Plasmodium.

Conclusions: Infections with A. lumbricoides, T. trichiura and S. mansoni were positively associated with P. falciparum infection. However, further studies are required to investigate how these helminths could contribute to increased prevalence of $P$. falciparum infection.

Keywords: Non-severe malaria, Association, Intestinal helminths, Plasmodium, Co-infections, Ethiopia

\footnotetext{
* Correspondence: abrahamdegarege@yahoo.com

Aklilu Lemma Institute of Pathobiology, Addis Ababa University, P.O. Box

1176, Addis Ababa, Ethiopia
}

\section{Ciomed Central




\section{Background}

Although Plasmodium and helminth co-infections are prevalent in tropical countries, the effect of their interactions remains unclear [1]. Some studies reported that helminth infected individuals are susceptible to Plasmodium infection [2-4], increased malaria gametocyte carriage [5], decreased heamoglobin concentration [6] and increased risk for clinical and severe malaria [7-9]. Crosssectional studies conducted in Zaire and Zimbabwe showed a significant positive association between the prevalence of $P$. falciparum malaria and intestinal helminths infection $[10,11]$. Positive association was also observed between Plasmodium density and intensity of hookworm infection [12].

On the other hand, evidence from other studies showed that helminth infections may protect from malaria or related clinical outcomes by suppressing acute clinical manifestations [13,14], parasite density in human blood $[15,16]$ or severe complications such as cerebral malaria, circulatory collapse, renal failure and jaundice [12,17-19].

Still other studies reported the absence of significant association between helminth infection and malaria $[3,20]$. These contradictory results could arise from the complex nature of the immune response to malaria parasites due to the variation in the Plasmodia stages and species or altered immune response because of helminth co-infections [1]. In addition, variations in study methodology, study design, case definition or malaria severity status, stage or intensity or species of helminths or Plasmodium, epidemiological setting of the study area and other confounding factors could contribute for the contradictions.

In Ethiopia, previous similar study was conducted in an area where infection with $P$. falciparum was less common (only $4.0 \%$ ), and infection with $S$. mansoni was absent [12]. In addition, in that previous study, intestinal helminth infections and haemoglobin concentration were determined only for Plasmodium infected cases, and factors such as age, sex, nutrition and socioeconomic status were not considered in the analysis. Thus, the current study was conducted to assess the nature of association of intestinal helminths with prevalence and clinical outcomes of Plasmodium infection among malaria suspected febrile individuals attending Dore Bafeno Health Center, Southern Ethiopia.

\section{Methods}

\section{Study area and participants}

A cross-sectional study involving 1,065 febrile patients (age range $=1$ to 82 years) was conducted at Dore Bafeno Health Center, Sidama Zone, southern Ethiopia, December 2010 to February 2011. The health center is located in Dore Bafeno District, about $283 \mathrm{~km}$ south of
Addis Ababa, at about $1,708 \mathrm{~m}$ above sea level and $7^{\circ} 5^{\prime} \mathrm{N}$ and $38^{\circ} 29 \mathrm{E}$. It has a mean annual rainfall of $80.4 \mathrm{~mm}$ (it varies from $15 \mathrm{~mm}$ to $130 \mathrm{~mm}$ ) and mean annual temperature of $23.2^{\circ} \mathrm{C}$ (it varies from $21.5^{\circ} \mathrm{C}$ to $25^{\circ} \mathrm{C}$ ). The study participants were malaria suspected febrile patients who did not take anti-malarial drug within the last two weeks. Almost all the study participants belonged to the Sidama ethnic group, residing in rural area with similar life style and earning their living as mixed-farming. Malaria is unstable in the area and occurs mainly from October to December following the heavy rainy season and from April to May following the light rain season (health facility reports).

\section{Socio-demographic and clinical data}

Clinical data including auxiliary body temperature, blood pressure, coma status, breathing status and prostration were collected by physicians. Clinical data together with laboratory results were used to group malaria cases as severe and non-severe [21]. Socio-demographic data including age, sex, height (to the nearest $0.1 \mathrm{~cm}$ ) and weight (to the nearest $0.1 \mathrm{~kg}$ ) were also recorded. Nutritional indicators (i.e. z-scores) were calculated using anthro [22] for children age $<5$ and using anthro-Plus [23] for children age between 5 and 19 years. Each child was classified as being under-nourished (if $\mathrm{z}$-score is less than -2) or well-nourished (if $z$-score is at least -2) [24]. For adults older than 19 years, the standard categories of body mass index (BMI $<18.5=$ undernourished; BMI $\geq 18.5$ well-nourished) were used to determine the nutritional status [25].

\section{Malaria microscopy and haemoglobin determination}

Approximately, $20 \mu \mathrm{l}$ finger prick blood was collected from each febrile patient using a plastic capillary tube and $10 \mu \mathrm{l}$ of the blood was used to prepare thick and thin blood films on a single slide for the diagnoses and count of malaria parasitaemia [26]. The slides were reexamined for quality assurance. The remaining blood sample was used to determine haemoglobin level using HemoCue HB 201, Anghelom, Sweden.

\section{Collection and examination of stool samples}

Fresh stool specimens were collected from each study participant who also gave blood sample for malaria diagnosis. The stool specimens were prepared for microscopic examination using double Kato slides [27]. Quantitative examination for hookworms was made within 45 minutes of stool collection, whereas T. trichiura, S. mansoni and A. lumbricoides infections were quantified at the Aklilu Lemma Institute of Pathobiology (ALIPB), Addis Ababa University. Average egg counts of the two slides was multiplied by 24 to obtain egg counts per gram of stool for each of the study participants and classes of intensity 
was determined for different helminth species as per the WHO recommendation [28].

\section{Ethical consideration}

This study was conducted after obtaining ethical clearance from the Institutional Review Board (IRB) of Aklilu Lemma Institute of Pathobiology, Addis Ababa University. As the majority of the population in the study area was illiterate the IRB endorsed oral consenting of the study participants. Permission to conduct the study was also obtained from Awassa Zuria District Health Office and Dore Bafeno Health Center. Detailed explanations about the aims, procedures, potential risks and benefit of the study were given to physicians of the health center. Individual oral consent was obtained in local language by the physicians of the health center during clinical examination and only voluntarily consented individuals were included in the study. For children younger than 18 years, assent was obtained from parents or guardians. Treatment was made based on the national protocol for treating malaria and helminth infections. Individuals who were found positive for $P$. falciparum and $P$. vivax infection were treated with coarteum and chloroquine, respectively. Individuals who were found positive for the soil-transmitted helminths (STHs) were treated with $400 \mathrm{mg}$ of albendazole while praziquantel was used to treat those who were positive for S. mansoni, Taenia saginata ( $T$. saginata), and Hymenolepis nana (H. nana). The study participants were treated free of charge. Since the study participants were selected from patients coming to the health center seeking medical treatment for malaria, they were not compensated for extra incentive to replace their transport cost or time lost from work was not given.

\section{Data analysis}

Data was computerized using Epi-data version 3.1 software (The EpiData Association, Odense, Denmark) and analyzed using STATA version 11 (Stata Corporation, College Station, Texas, USA). Pearson $x^{2}$-test and oneway ANOVA were used to compare malaria prevalence and mean parasitaemia or egg intensity values, respectively across categories of categorical variables. Univariable and multivariable logistic regression analyses were used to test for an association between intestinal helminths infection and prevalence of non-severe malaria or malaria related anaemia. Multiple linear regression analyses was used to evaluate differences in the means of Plasmodium density and haemoglobin level between Plasmodium-helminth co infected and those who were infected with Plasmodium alone. The analysis was adjusted for age, sex and nutritional status [29,30], while the association between helminth and Plasmodium infections, or related outcomes was evaluated. Since the study population was homogenous in their socio-economic status differences in terms of socioeconomic status were not considered during the analysis. The distribution of parasite density was skewed and square root transformation was used to make it symmetrical before using linear regression to evaluate the effect of pre-specified risk factors on this outcome. Results were considered significant whenever p-value was less than $5 \%$.

\section{Results}

\section{Prevalence of malaria}

Among 1,065 malaria suspected febrile patients (Mean age $=18.6$ years), 306 (28.8\%) were positive for Plasmodium parasites (Table 1). Plasmodium infection was higher among febrile patients in the 5-15 years age group and in the under 5 children compared to individuals older than 15 years $(p<0.001)$. P. falciparum infection alone or mixed infection with $P$. vivax was also higher in the 5-15 years old children compared to in individuals older than 15 years $(p<0.01)$. Infection with $P$. vivax alone was higher $(p<0.001)$ in the under five children and in the 5-15 years age group compared to those individuals older than 15 years. There was no significant difference in the percentage of $P$. falciparum and/or $P$. vivax infection between males and females.

\section{Prevalence of helminths infection}

Among 1,065 febrile patients examined for malaria, 702 were volunteered to provide stool samples for the diagnosis of infection with intestinal helminths. There was no significant difference in baseline demographics between those who provided stool sample and those who did not (data not shown). Out of 702 patients examined, $53.8 \%$ were positive for at least one intestinal helminth species (Table 2). The diagnosed helminth species were A. lumbricoides (35.9\%), T. trichiura (15.8\%), S. mansoni (11.7\%), hookworm (9.8\%), T. saginata (3.9\%), H. nana (1.4\%) and Enterobius vermicularis (E. vermicularis) $(0.9 \%)$. Of the 378 intestinal helminth infected individuals, $35.8 \%, 13.3 \%, 4.3 \%$ and $0.6 \%$ had single, double, triple and quadruple infections, respectively. Intestinal helminth infection was most prevalent among individuals of age 5-15 years (63.2\%). A. lumbricoides and T. trichiura infections were higher among individuals in 5-15 years age group than among individuals of age $>15$ years.

Mean intensity of infections were 2345 egg per gram \{epg\} (range; 24-16128) for A. lumbricoides, 372 epg (range; 24-12816) for S. mansoni, 107 epg (range; 24-1200) for hookworm and 101 epg (range; 24-1296) for T. trichiura. Intensity of $A$. lumbricoides infection showed age related pattern with the highest $(\mathrm{F}=3.36, p=0.036)$ mean intensity being observed among individuals of age group $5-15$ years (mean epg=3113.7) followed by individuals of 
Table 1 Prevalence of malaria among malaria suspected febrile patients who attended Dore Bafeno Health Center, southern Ethiopia, December 2010

\begin{tabular}{|c|c|c|c|c|c|}
\hline Variables & $\begin{array}{l}\text { Number } \\
\text { examined }\end{array}$ & $\begin{array}{l}\text { P.falciparum } \\
\text { positive } \mathrm{n}(\%)\end{array}$ & $\begin{array}{l}\text { P. vivax positive } \\
\mathrm{n}(\%)\end{array}$ & $\begin{array}{l}P \text {. falciparum \& } P \text {. vivax } \\
\text { positive } n(\%)\end{array}$ & $\begin{array}{l}\text { Total malaria } \\
\text { positive } \\
\mathrm{n}(\%)\end{array}$ \\
\hline \multicolumn{6}{|l|}{ Age } \\
\hline$<5$ & 255 & $28(11.0)$ & $57(22.4)$ & $3(1.2)$ & $88(34.5)$ \\
\hline $5-15$ & 240 & $56(23.3)$ & 39 (16.3) & $7(2.9)$ & $102(42.5)$ \\
\hline$>15$ & 570 & $54(9.5)$ & $58(10.2)$ & $4(0.7)$ & $116(20.4)$ \\
\hline$x^{2}(p)$ & & $36.12(<0.001)$ & $26.35(<0.001)$ & $9.69(0.008)$ & $45.91(<0.001)$ \\
\hline \multicolumn{6}{|l|}{ Sex } \\
\hline Female & 522 & $64(12.3)$ & 68 (13.0) & $8(1.5)$ & $140(26.8)$ \\
\hline Male & 543 & 74 (13.6) & 86 (15.8) & $6(1.1)$ & $166(30.6)$ \\
\hline$x^{2}(p)$ & & $0.73(0.393)$ & $1.95(0.162)$ & $0.26(0.613)$ & $1.83(0.176)$ \\
\hline
\end{tabular}

age group $<5$ years (mean epg=2410.6). Mean intensity of hookworm infection was higher $(\mathrm{F}=15.69, p<0.001)$ among individuals of age $<5$ years (mean epg=648) followed by those individuals whose age ranges from $5-15$ years $($ mean epg $=69.6)$.

\section{Malaria and helminth co-infection}

Out of 702 patients, 242 (34.5\%) were infected with intestinal helminths alone, 86(12.3\%) with Plasmodium alone, and 136 (19.4\%) with both Plasmodium and intestinal helminths (Table 3). Intestinal helminthic infection was significantly associated with increased odds of malarial infection (i.e. P. falciparum and/or $P$. vivax infection) (unadjusted $\mathrm{OR}=1.56,95 \% \mathrm{CI}=1.13-2.16$, $p=0.007)$. Prevalence of $P$. falciparum infection was significantly higher in individuals infected with any intestinal helminth (s), A. lumbricoides alone, T. trichiura alone, $S$. mansoni alone compared to those who were not infected with intestinal helminths $(9.3 \%) \quad(p<0.001$ for all) (Table 3).
Out of 306 Plasmodium infected patients, 14 showed one or more sign and symptoms of severe malaria (i.e. prostration in 10 patients, breathing difficulty syndrome in 8 patients, cerebral malaria in 7 patients and severe anaemia in 3 patients). Nine severe malaria cases were volunteered to provide stool samples for the diagnosis of helminth infection and 7 of them were co-infected with intestinal helminths like $A$. lumbricoides, $T$. trichiura and hook worm.

Of the 292 non-severe malaria cases, 213 patients were examined for intestinal helminth infection and $61.0 \%$ were positive. The odds of non-severe malaria infection was higher in individuals infected with intestinal helminth compared to intestinal helminth-free individuals \{adjusted Odds Ratio $(\mathrm{AOR})=1.57,95 \% \mathrm{CI}=1.12,2.21$. Similarly, the odds of non-severe $P$. falciparum monoinfection was higher among individuals infected with intestinal helminth than non-infected with intestinal helminth $(\mathrm{AOR}=2.89,95 \% \mathrm{CI}=1.77,4.72)$. The ORs changed in parallel with the number of intestinal helminth species infection $(\mathrm{p}<0.001)$. Individuals infected

Table 2 Intestinal helminth infection among malaria suspected febrile patients Dore Bafeno Health Center, southern Ethiopia, December 2010

\begin{tabular}{|c|c|c|c|c|c|c|c|}
\hline Variable & $\begin{array}{l}\text { Number } \\
\text { examined }\end{array}$ & $\begin{array}{l}\text { A.lumbricoides } \\
\text { n (\%) }\end{array}$ & $\begin{array}{l}\text { Hookworm } \\
\text { n (\%) }\end{array}$ & $\begin{array}{l}\text { T. trichiura } \\
\text { n (\%) }\end{array}$ & $\begin{array}{l}\text { S. mansoni } \\
\mathrm{n}(\%)\end{array}$ & $\begin{array}{l}\text { Others* } \\
\text { n (\%) }\end{array}$ & $\begin{array}{l}\text { Any intestinal } \\
\text { helminth infection** } \\
n(\%)\end{array}$ \\
\hline \multicolumn{8}{|l|}{ Age } \\
\hline$<5$ & 88 & $24(27.3)$ & $2(2.3)$ & 12 (13.6) & $3(3.4)$ & $6(6.8)$ & 35 (39.3) \\
\hline $5-15$ & 182 & $86(47.3)$ & $10(5.5)$ & $36(19.8)$ & $24(13.2)$ & $9(5.0)$ & $115(63.2)$ \\
\hline$>15$ & 432 & $142(32.9)$ & $57(13.2)$ & 63 (14.6) & $55(12.7)$ & $15(3.5)$ & $228(52.8)$ \\
\hline$x^{2}(p)$ & & $14.76(0.001)$ & $15.05(0.001)$ & $2.96(0.228)$ & $6.70(0.035)$ & $5.96(0.202)$ & $14.13(0.001)$ \\
\hline \multicolumn{8}{|l|}{ Sex } \\
\hline Female & 341 & 135 (39.6) & $28(8.2)$ & 65 (19.1) & $33(9.7)$ & $19(5.6)$ & $190(55.72)$ \\
\hline Male & 361 & $117(32.4)$ & $41(11.4)$ & 46 (12.7) & 49 (13.6) & $11(3.0)$ & 188 (51.93) \\
\hline$x^{2}(p)$ & & $3.93(0.047)$ & $1.96(0.162)$ & $5.26(0.022)$ & $2.58(0.108)$ & $3.06(0.217)$ & $1.02(0.314)$ \\
\hline
\end{tabular}

Others* ${ }^{*}$ infection with either of $H$. nana, $T$. Saginata, E. vermucularis.

Any intestinal helminth infection ${ }^{* *}=$ infection with at least one intestinal helminth species. 
Table 3 Prevalence of malaria and intestinal helminth co-infections among malaria suspected febrile patients who attended Dore Bafeno Health Center, southern Ethiopia, December 2010

\begin{tabular}{|c|c|c|c|c|c|}
\hline Helminth infection & $\begin{array}{l}\text { Number } \\
\text { of cases }\end{array}$ & $\begin{array}{l}\text { P. falciparum } \\
\text { positive } \mathrm{n}(\%)\end{array}$ & $\begin{array}{l}P \text {. vivax } \\
\text { positive } \mathrm{n}(\%)\end{array}$ & $\begin{array}{l}\text { P. falciparum and } \\
\text { P. vivax } \mathrm{n}(\%)\end{array}$ & $\begin{array}{r}\text { Total malaria } \\
\text { positive } \mathrm{n}(\%)\end{array}$ \\
\hline Only A. lumbricoides (A) & 141 & $30(21.3)$ & $20(14.2)$ & $2(1.4)$ & $52(36.9)$ \\
\hline Only hookworm (Hw) & 25 & $1(4.0)$ & $6(24.0)$ & 0 & $7(28.0)$ \\
\hline Only T. trichiura $(T t)$ & 39 & $9(23.1)$ & $6(15.4)$ & 0 & $15(38.5)$ \\
\hline Only S. mansoni (Sm) & 39 & $9(23.1)$ & $3(7.7)$ & 0 & $15(38.5)$ \\
\hline$A /$ and $T t$ & 42 & $9(31.0)$ & $11(35.5)$ & $2(4.5)$ & $22(52.4)$ \\
\hline$A l$ and $S m$ & 18 & $4(22.2)$ & $1(5.6)$ & 0 & $5(27.8)$ \\
\hline$A l, T t$ and $S m$ & 5 & $1(20.0)$ & $1(20.0)$ & 0 & $2(40.0)$ \\
\hline$A l, T t, S m$ and $H w$ & 22 & $7(31.8)$ & $1(4.5)$ & 0 & $8(36.4)$ \\
\hline Any intestinal helminth & 378 & $80(21.2)$ & $51(13.5)$ & $5(1.3)$ & $136(36.0)$ \\
\hline Without intestinal helminths & 324 & $30(9.3)$ & $50(15.4)$ & $6(1.9)$ & $86(26.5)$ \\
\hline
\end{tabular}

with A. lumbricoides alone (AOR=2.6), T. trichiura alone $(\mathrm{AOR}=2.8)$, S. mansoni alone $(\mathrm{AOR}=3.3)$, any intestinal helminth $(\mathrm{AOR}=2.9), T$. trichiura and A. lumbricoides (AOR=3.1), and T. trichiura, A. lumbricoides, S. mansoni and hookworm $(\mathrm{AOR}=4.0)$ had increased odds of nonsevere $P$. falciparum mono-infection compared to those who were not infected with any intestinal helminths $(p<0.05$ for all) (Table 4). However, the proportions of $P$. vivax mono-infected individuals were not significantly different when compared between those who had single or multiple infections with different intestinal helminths and intestinal helminth free individuals (data not shown). Similarly, infection with hookworm alone was not significantly associated with any of non-severe $P$. falciparum and/or P. vivax infection.

Data regarding the effect of intestinal helminths co infection on malaria-related outcomes are summarized in Table 5. After adjusting for age, sex and nutritional status mean Plasmodium density was significantly higher in individuals co-infected with four different intestinal helminth species (T. trichiura, A. lumbricoides, S. mansoni and hookworm) compared to those who were free from

Table 4 Association of intestinal helminth infection and non-severe $\boldsymbol{P}$. falciparum malaria among malaria suspected febrile patients who attended Dore Bafeno Health Center, southern Ethiopia, December 2010

\begin{tabular}{|c|c|c|c|c|c|}
\hline Outcome & Main exposure & Model & OR & $95 \% \mathrm{Cl}$ & p-value \\
\hline \multirow[t]{18}{*}{ P. falciparum } & Only A. lumbricoides (A) & Crude & 2.58 & $1.44,4.61$ & 0.001 \\
\hline & & Adjusted* & 2.55 & $1.40,4.63$ & 0.002 \\
\hline & Only hookworm (Hw) & Crude & 0.47 & $0.06,3.67$ & 0.473 \\
\hline & & Adjusted* & 0.54 & $0.07,4.35$ & 0.565 \\
\hline & Only $T$. trichiura $(T t)$ & Crude & 2.48 & $0.98,6.27$ & 0.055 \\
\hline & & Adjusted* & 2.77 & $1.15,6.65$ & 0.023 \\
\hline & Only S. mansoni (Sm) & Crude & 2.83 & $1.21,6.63$ & 0.016 \\
\hline & & Adjusted* & 3.25 & $1.32,7.97$ & 0.010 \\
\hline & $A l$ and $T t$ & Crude & 3.57 & $1.49,8.55$ & 0.004 \\
\hline & & Adjusted* & 3.12 & $1.27,7.61$ & 0.013 \\
\hline & $A l$ and $S m$ & Crude & 2.44 & $0.75,7.96$ & 0.139 \\
\hline & & Adjusted* & 2.65 & $0.76,9.22$ & 0.125 \\
\hline & $A l, T t$ and $S m$ & Crude & 2.64 & $0.27,26.23$ & 0.406 \\
\hline & & Adjusted* $^{*}$ & 2.34 & $0.23,23.88$ & 0.473 \\
\hline & $A l, T t, S m$ and $H w$ & Crude & 3.96 & $1.48,10.60$ & 0.006 \\
\hline & & Adjusted* & 4.12 & $1.49,11.31$ & 0.006 \\
\hline & Any intestinal helminth & Crude & 2.74 & $1.71,4.41$ & 0.000 \\
\hline & & Adjusted* & 2.89 & $1.77,4.72$ & 0.000 \\
\hline
\end{tabular}

NB 1. Individuals free from any intestinal helminth infection were used as reference categories while calculating the OR.

2. Adjusted ${ }^{*}=$ the model is adjusted for the effects of age, gender and nutritional status.

3. Any intestinal helminth = individuals infected with at least 1 intestinal helminth species. 
Table 5 Effect of intestinal helminth infection on malaria related outcomes among microscopic confirmed malaria cases who attended Dore Bafeno Health Center, southern Ethiopia, December 2010 to February 2011

\begin{tabular}{|c|c|c|c|}
\hline \multirow{3}{*}{ Helminth infection } & \multirow{3}{*}{$\frac{\text { Plasmodium }}{=\beta^{* * *}[95 \% \mathrm{Cl}]}$} & \multirow{2}{*}{$\frac{\text { Outcomes }}{\text { Haemoglobin }}$} & \multirow[b]{2}{*}{ Anaemia } \\
\hline & & & \\
\hline & & $\beta * * *[95 \% \mathrm{Cl}]$ & $\mathrm{AOR}^{* *}[95 \% \mathrm{Cl}]$ \\
\hline A. lumbricoides (Al) & $2.34[-24.68,20.00]$ & $-0.52[-1.37,0.32]$ & $1.53[0.72,3.22]$ \\
\hline Hookworm (Hw) & $-11.75[-83.76,60.26]$ & $-0.65[-2.64,1.34]$ & $1.69[0.33,8.81]$ \\
\hline S. mansoni (Sm) & $3.65[-35.09,42.41]$ & $0.12[-1.45,1.68]$ & $1.11[0.30,4.17]$ \\
\hline T. trichiura $(T t)$ & $19.11[-13.31,51.55]$ & $-0.65[-2.64,1.34]$ & $1.69[0.33,8.81]$ \\
\hline$A /$ and $T t$ & $15.16[-21.34,51.67]$ & $-.63[-1.77,0.50]$ & $2.31[0.80,6.67]$ \\
\hline$A l$ and $S m$ & $22.19[-55.82,100.19]$ & $-.08[-2.68,2.52]$ & $1.27[0.17,9.47]$ \\
\hline$A l, T t$ and $S m$ & $64.65[-10.53,139.85]$ & $-2.35[-6.35,1.63]$ & $2.83[0.98,15.24]$ \\
\hline$A l, T t, S m$ and $H w$ & $52.05[5.08,99.03]$ & $-1.62[-3.61,0.37]$ & $8.64[1.18,63.47]$ \\
\hline Any intestinal helminth & $14.51[-3.06,32.07]$ & $-0.34[-0.99,0.30]$ & $1.54[0.81,2.90]$ \\
\hline
\end{tabular}

NB 1.helmith free but Plasmodium infected individuals are used as reference for calculating AOR-values and regression coefficients.

2. Plasmodium density* ${ }^{*}$ Plasmodium density is on square root scale.

3. $A R^{* *}$ odds ratio from multivariate logistic regression models adjusted for effects of age, gender and nutritional status.

4. $\beta$ *** regression coefficient from multiple linear regression models adjusted for effects of age, gender and nutrition.

intestinal helminth infection $(\beta=10829.56, p<0.05)$. Similarly, there was an increasing trend in mean Plasmodium parasitaemia among individuals who were infected with $T$. trichiura alone, S. mansoni alone, A. lumbricoides alone and any two or three of these species compared to individuals who were not infected with any intestinal helminth species. The mean Plasmodium density among intestinal helminth infected individuals increased significantly as the number of intestinal helminth species increased $(\mathrm{F}=2.82, P=0.027)$. However, patients infected with hookworm alone showed slightly lower mean Plasmodium parasitaemia compared to individuals who had no intestinal helminth infection.

Individuals co-infected with $A$. lumbricoides, hookworm, S. mansoni, T. trichiura alone or in combination and Plasmodium showed lower mean haemoglobin levels compared to malaria cases without intestinal helminths infections. However, the mean differences were not significant in the multivariable model. Prevalence of anaemia was higher among individuals co-infected with both intestinal helminths and Plasmodium compared to those with Plasmodium infection alone. Individuals coinfected with Plasmodium and four different intestinal helminths were almost 9 times more likely to be anaemic compared to those with Plasmodium infection alone (AOR=8.64, 95\% $\mathrm{CI}=1.18,63.47)$.

There was not significant correlation between the mean Plasmodium density and intensity of intestinal helminth infections (spearman correlation coefficient= $0.12,-0.19,0.04,-0.08$ for A. lumbricoides, hookworm, T. trichiura, S. mansoni, respectively). In S. mansoni and T. trichiura infected patients, the mean parasitaemia increased as the class of intensity of the helminths increased; nonetheless the regression coefficients remain non-significant after adjusting for the effects of age, sex, nutrition, and concurrent infection by other species of helminth. Conversely, the mean parasitaemia of individuals with moderate intensity of $A$. lumbricoides infection was relatively low compared to individuals co infected with light intensity of $A$. lumbricoides and Plasmodium. For hookworm infection, all the infected cases were of light intensity class (data not shown).

The reduction in mean haemoglobin level among individuals co-infected with intestinal helminths and Plasmodium was negatively correlated with intensity of intestinal helminths. However, the coefficients for the association were low. Similarly, although the mean differences were not significant, haemoglobin levels decreased more in Plasmodium infected individuals co-infected with heavy intense $S$. mansoni or moderate intense T. trichiura infections compared to individuals with light intensity of infections with the respective intestinal helminths (data not shown).

\section{Discussion}

In this study, the likelihood of being infected with nonsevere $P$. falciparum malaria was significantly higher in individuals infected with intestinal helminths, particularly in those with $A$. lumbricoides alone, $T$. trichiura alone or $S$. mansoni alone compared to individuals without intestinal helminths. Similarly, prevalence of non-severe malaria (P. falciparum and/or P. vivax) was significantly higher in individuals infected with intestinal helminth or A. lumbricoides alone compared to individuals without intestinal helminths. These findings are similar with previous finding in children $[9,11]$ and adults [10]. Increased incidence of $P$. falciparum malaria was also previously reported in helminth infected individuals $[3,7,8]$. 
In concordance with previous reports [6,31], a relatively lower mean heamoglobin level was observed among Plasmodium and intestinal helminth co-infected patients compared to those with only Plasmodium infection. There was a non-significant decreasing trend in mean haemoglobin levels as the number of intestinal helminths species per an individual increased. Similarly, the association of anaemia was slightly higher in individuals co-infected with Plasmodium and intestinal helminth compared to malaria cases without having intestinal helminth infections. Intestinal helminths and malaria reduce haemoglobin level differently [32,33], implying that their effect will be additive in causing anaemia when they coexist in a host.

Mean Plasmodium density was slightly higher among intestinal helminth infected individuals compared with intestinal helminth free individuals. These results together with the high odds of anaemia (or decreased mean haemoglobin levels) and non-severe P. falciparum and/or $P$. vivax infection give the impression of a positive association between intestinal helminth infection and non-severe malaria. This association between prevalence or outcomes of malaria and intestinal helminth infections seemed to increase with an increase in the number of intestinal helminths species. This is in agreement with previous reports from Ethiopia [12] and Thailand [3] where the risk of high Plasmodium density and P. falciparum infection, respectively increased with the number of intestinal helminth species.

The present findings appear to substantiate the hypothesis that clinical malaria can be aggravated during concomitant infection of intestinal helminths and Plasmodium [32]. Worms are known to induce regulatory $\mathrm{T}$ cells leading to the production of cytokines that counteracts Th1 response [34]. Helminth also modulates the function of denderitic cells, consequently altering the immune response to malaria antigen [1]. Additionally, helminth ligand repeatedly stimulate toll like receptors, thereby down regulating responsiveness to Plasmodium during concomitant infection [35]. Helminth infection could also make the skin less retort to mosquito bites [36] promoting the success of sporozoite to pass through it and increasing the chance of blood stage infection.

However, Plasmodium density was less associated with intensity of helminth infections and results regarding mean differences of Plasmodium density between intestinal helminth free and those infected with different intestinal helminth species were contradictory. Compared to intestinal helminth free malaria cases, the mean Plasmodium density of individuals infected with A. lumbricoides alone, S. mansoni alone or T. trichiura alone was slightly higher, but lower in the case of single hookworm infection. These controversial results on mean Plasmodium changes could have arisen due to the dominancy of light intense helminth infection among cases or complex nature of helminth and Plasmodium interaction when co-exist in a host.

Individuals with hookworm infection alone were neither protected nor associated with increased non-severe $P$. falciparum and/or P. vivax infection. This is in agreement with previous reports from Uganda [20] and Zaire [37], but in conflict with those from Zimbabwe [11]. The low infection intensity of hookworm (all cases light infection) in the present study might have contributed to the present finding. Had some of the cases been with moderate and/or heavy intensity classes the nature of the association could have been better explored. In addition, the present study revealed the absence of significant association between $P$. vivax mono-infection and infection with different intestinal helminth species which agrees with the observation in Thailand [18]. This could be due to the difference in pre-erythrocyte immunity for the two Plasmodium species [38].

Previous studies documented negative [3,17-19] and positive [8] association between geohelminth infection and malaria severity. However, the number of severe malaria cases in the current study was very low, limiting assessment about the nature of association between intestinal helminth infection and complicated malaria.

Variation in inferences about the nature of malaria and helminth interaction among reports could be due to differences in the nature of the study participants (children, pregnant, adults), study design, methodology and the way data was analyzed and interpreted. In most previous studies, data analysis did not separate the effect of different helminth or Plasmodium species. In addition, the effect of confounding factors such as age, sex, nutrition status, socioeconomic factors and other infections (e.g., HIV) were not usually considered in previous studies. It is well known that, level of endemicity (both helminths and malaria), local climate, traditional and modern health care practices as well as community health seeking behaviors vary from country to country and even from district to district. These variations could also bring substantial differences in helminth-malaria interactions and hence a result from one country/district might not be necessarily generalized or interpreted in another country/district where the above factors are significantly different.

Participants of the current study were more homogenous in that they were largely $(>90 \%$ of the cases) similar in their life style, education level (illiterate), income level (average) and means of making a living (agriculture), place of residence (rural) and ethnicity (Sidama). However, slight differences in socioeconomic status might have existed among the cases which could partly affect the current data. In addition, cross-sectional nature of the study design has been a limitation to make 
conclusion about the risk or incidence of malaria due to previous status of helminth infection.

\section{Conclusions}

In the current study, infections with A. lumbricoides, T. trichiura and S. mansoni were positively associated with $P$. falciparum infection. However, further studies are required to investigate how these helminths could contribute to increased prevalence of $P$. falciparum infection.

\section{Competing interests}

The authors declare they have no competing interests.

\section{Authors' contributions}

AD: conceived the project idea and designed the study, collected, analyzed and interpreted the data. GM: involved in data analysis. AD, GM, ML, AA and $\mathrm{BE}$ involved in the drafting of the manuscript for important intellectual content. All authors read the draft manuscript and approved the final copy for submission.

\section{Acknowledgements}

This study received financial support from Aklilu Lemma Institute of Pathobiology, Addis Ababa University. We would like to thank the staffs of Dore Bafeno Heath Center for their cooperation during the study. We are also grateful to the study participants without whom the current study would not have been realized.

Received: 14 November 2011 Accepted: 3 November 2012

Published: 9 November 2012

\section{References}

1. Hartgers FC, Yazdanbakhsh M: Co-infection of helminths and malaria: modulation of the immune responses to malaria. Parasite Immunol 2006 28:497-506

2. Nacher M, Singhasivanon P, Gay F, Silachomroon U, Phumratanaprapin W, Looareesuwan S: Contemporaneous and successive mixed Plasmodium falciparum and Plasmodium vivax infections are associated with Ascaris lumbricoides: an immunomodulating effect? J Parasitol 2001, 87:912-915.

3. Nacher $M$, Singhasivanon $P$, Yimsamran $S$, Manibunyong $W$, Thanyavanich N, Wuthisen R, Looareesuwan S: Intestinal helminth infections are associated with increased incidence of Plasmodium falciparum malaria in Thailand. J Parasitol 2002, 88:55-58.

4. Chaorattanakawee S, Natalang O, Hananantachai H, Nacher M, Brockman A, Nosten F, Looareesuwan S, Patarapotikul J: Trichuris trichiura infection is associated with the multiplicity of Plasmodium falciparum infections, in Thailand. Ann Trop Med Parasitol 2003, 97:199-202.

5. Nacher M, Singhasivanon P, Silachamroon U, Treeprasertsu S, Krudsood S, Gay F, Mazier D, Looareesuwan S: Association of helminth infections with increased gametocyte carriage during mild falciparum malaria in Thailand. Am J Trop Med Hyg 2001, 65:644-647.

6. Nacher M, Singhasivanon P, Gay F, Phumratanaprapin W, Silachomroon U, Looareesuwan S: Association of helminth infection with decreased reticulocyte counts and haemoglobin concentration in Thai Falciparum malaria. Am J Trop Med Hyg 2001, 65:335-337.

7. Spiegel A, Tall A, Raphenon G, Trape JF, Druilhe P: Increased frequency of malaria attacks in subjects co-infected by intestinal worms and Plasmodium falciparum malaria. Trans R Soc Trop Med Hyg 2003, 97:198-199.

8. Le Hesran JY, Akiana J, Ndiayeel HM, Dia M, Senghor P, Konate L: Severe malaria attack is associated with high prevalence of Ascaris lumbricoides infection among children in rural Senegal. Trans R Soc Trop Med Hyg 2004, 98:397-399.

9. Sokhna C, Le Hesran JY, Mbaye PA, Akiana J, Camara P, Diop M, Ly A, Druilhe $P$ : Increase of malaria attacks among children presenting concomitant infection by Schistosoma mansoni in Senegal. Malar J 2004, 3:43.
10. Tshikuka JG, Scott ME, Gray-Donald K, Kalumba ON: Multiple infection with Plasmodium and helminth in communities of low and relatively high socio-economic status. Ann Trop Med Parasitol 1996, 90:277-293.

11. Midzi N, Sangweme D, Zinyowera S, Mapingure MP, Brouwer KC, Munatsi A, Mutapi F, Mudzori J, Kumar N, Woelk G, Mduluza T: The burden of polyparasitism among primary schoolchildren in rural and farming areas in Zimbabwe. Trans R Soc Trop Med Hyg 2008, 102:1039-1045.

12. Degarege $A$, Animut $A$, Legesse $M$, Erko B: Malaria severity status in patients with soil-transmitted helminth infections. Acta Trop 2009, 112:8-11.

13. Murray MJ, Murray AB, Murray MB, Murray CJ: Parotid enlargement, forehead edema, and suppression of malaria as nutritional consequences of ascariasis. Am J Clin Nutr 1977, 30:2117-2121.

14. Murray J, Murray A, Murray M, Murray C: The biological suppression of malaria: an ecological and nutritional interrelationship of a host and two parasites. Am J Clin Nutr 1978, 31:1363-1366.

15. Brutus $L$, Watier $L$, Briand $V$, Hanitrasoamampionona $V$, Razanatsoarilala $H$, Cot M: Parasitic co-infections: does Ascaris lumbricoides protect against Plasmodium falciparum infection? Am J Trop Med Hyg 2006, 75:194-198.

16. Brutus L, Watier L, Hanitrasoamampionona V, Razanatsoarilala H, Cot M: Confirmation of the protective effect of Ascaris lumbricoides on Plasmodium falciparum infection: results of a randomized trial in Madagascar. Am J Trop Med Hyg 2007, 77:1091-1095.

17. Nacher M, Gay F, Singhasivanon P, Krudsood S, Treeprasertsuk S, Mazier D, Vouldoukis I, Looareesuwan S: Ascaris lumbricoides infection is associated with protection from cerebral malaria. Parasite Immunol 2000, 22:107-113.

18. Nacher M, Singhasivanon P, Traore B, Vannaphan S, Gay F, Chindanond D, Franetich JF, Mazier D, Looareesuwan S: Helminth infections are associated with protection from cerebral malaria and increased nitrogen derivatives concentrations in Thailand. Am J Trop Med Hyg 2002 66:304-309.

19. Nacher M, Singhasivanon $P$, Silachamroon U, Treeprasertsuk S, Vannaphan S, Traore B, Gay F, Looareesuwan S: Helminth infections are associated with protection from malaria-related acute renal failure and jaundice in Thailand. Am J Trop Med Hyg 2001, 65:834-836.

20. Shapiro AE, Tukahebwa EM, Kasten J, Clarke SE, Magnuseen P, Olsen A, Kabatereine NB, Ndyomugyenyi R, Brooker S: Epidemiology of helminth infections and their relationship to clinical malaria in southwest Uganda. Trans R Soc Trop Med Hyg 2005, 99:18-24.

21. World Health Organization: Severe falciparum malaria. Trans $R$ Soc Trop Med Hyg 2000, 94:1-90.

22. World Health Organization: Anthro for personal computers, version 3.1, 2010: Software for assessing growth and development of the world's children. Geneva: WHO; 2010.

23. World Health Organization: AnthroPlus for personal computers Manual: Software for assessing growth of the world's children and adolescents. Geneva: WHO; 2009

24. World Health Organization: Child Growth Standards: length /height-for-age, weight-for-age, weight-for-length, weight-for height and body mass index-forage. Methods and development. Geneva: WHO; 2006.

25. World Health Organization: Physical status: the use and interpretation of anthropometry. Expert Committee Report. WHO Technical Report Series 1995, 854:1-460. Geneva.

26. Cheesbrough M: Parasitological Tests, District Laboratory Practices in Tropical Countries. England: Part I. Cambridge University Press; 1998:220-221.

27. World Health Organization: Basic Laboratory Methods in Medical Parasitology. Geneva: WHO; 1991.

28. WHO: Prevention and control of schistosomiasis and soil-transmitted helminthiasis. Tech Rep Ser 2002, 912:1-57.

29. Custodio E, Descalzo MA, Villamor E, Molina L, Sánchez I, Lwanga M, Bernis C, Benito A, Roche J: Nutritional and socio-economic factors associated with Plasmodium falciparum infection in children from Equatorial Guinea: results from a nationally representative survey. Malar J 2009, 8:225.

30. Quihui L, Valencia ME, Crompton DW, Phillips S, Hagan P, Morales G, Díaz-Camacho SP: Role of the employment status and education of mothers in the prevalence of intestinal parasitic infections in Mexican rural schoolchildren. BMC Public Health 2006, 6:225.

31. Degarege A, Animut A, Legesse M, Erko B: Malaria and helminth coinfections in outpatients of Alaba Kulito Health Center, southern Ethiopia: a cross sectional study. BMC Research Notes 2010, 3:143-149. 
32. Mwangi TW, Bethony JM, Brooker S: Malaria and helminth interactions in humans: an epidemiological viewpoint. Ann Trop Med Parasitol 2006, 100:551-570.

33. Brooker S, Akhwale W, Pullan R, Estambale B, Clarke SE, Snow RW, Hotez JP: Epidemiology of plasmodium-helminth co-infection in Africa: populations at risk, potential impact on anemia, and prospects for combining control. Am J Trop Med Hyg 2007, 77:88-98.

34. Maizels RM, Yazdanbakhsh M: Immune regulation by helminth parasites: cellular and molecular mechanisms. Nat Rev Immunol 2003, 3:733-744.

35. Boutlis CS, Yeo TW, Anstey NM: Malaria tolerance-for whom the cell tolls? Trends Parasitol 2006, 22:371-377.

36. Hagel I, Lynch NR, Pérez M, Di Prisco MC, López R, Rojas E: Modulation of the allergic reactivity of slum children by helminthic infection. Parasite Immunol 1993, 15:311-315.

37. Mazigo HD, Waihenya R, Lwambo NJS, Mnyone LL, Mahande AM, Seni J, Zinga M, Kapesa A, Kweka EJ, Mshana SE, Heukelbach J, Mkoji GM: Co-infections with Plasmodium falciparum. Schistosoma mansoni and intestinal helminths among schoolchildren in endemic areas of northwestern Tanzania. Parasites \& Vectors 2010, 3:44.

38. Luxemburger C, Thwai KL, White NJ, Webster HK, Kyle DE, Maelankirri L, Chongsuphajaisiddhi T, Nosten F: The epidemiology of malaria in a Karen population on the western border of Thailand. Trans R Soc Trop Med Hyg 1996, 90:105-111.

doi:10.1186/1471-2334-12-291

Cite this article as: Degarege et al:: Malaria and related outcomes in

patients with intestinal helminths: a cross-sectional study. BMC Infectious Diseases 2012 12:291.

\section{Submit your next manuscript to BioMed Central and take full advantage of:}

- Convenient online submission

- Thorough peer review

- No space constraints or color figure charges

- Immediate publication on acceptance

- Inclusion in PubMed, CAS, Scopus and Google Scholar

- Research which is freely available for redistribution 